\title{
Endothelial damage and thrombotic response in patients with cured Cushing Syndrome
}

Gloria Aranda 1,2, Mireia Mora 1,2, Irene Halperin 1,2, Rebeca Fernández², Maribel Diaz-Ricart ${ }^{3}$, Marta Palomo³, Oscar Vidal4,2, Joaquim Enseñat ${ }^{5}$, Gregori Casals $^{6}$, Felicia Alexandra Hanzu ${ }^{1,2}$.

1. Endocrinology Department, HCB/IDIBAPS/CIBERDEM, Barcelona, Spain. 2. Endocrine Disorder Laboratory IDIBAPS/CIBERDEM, Barcelona, Spain. 3. Hemostasy Laboratory, HCB/IDIBAPS, Barcelona, Spain. ${ }^{4}$ Endocrine Surgery, HCB/IDIBAPS, Barcelona, Spain. ${ }^{5}$ Neurosurgery Department, HCB/IDIBAPS, Barcelona, Spain. ${ }^{6}$ Hormonal Laboratory, HCB/IDIBAPS, Barcelona, Spain.

\section{Background}

Clinical observational studies have reported the persistence of a high cardiovascular risk in patients with cured Cushing's Syndrome (CCS) compared with controls of the same age, gender and body mass index (BMI). It is still at debated whether this is due to the persistence of comorbidities, hormone deficiencies or chronic changes induced by hypercortisolism.

\section{Aim}

To investigate the interplay in CCS of the cardiovascular disease in vivo and the endothelial activation and thrombogenicity in vitro occurring in response to sera of CCS.

\section{Methods}

Cross-sectional study in CS patients and controls and in vitro endothelial damage atherothrombotic model.

Subjects:

I. CCS (n:10) at minimun 2 years after cure and without hormone deficiencies.

II. Active CS (ACS) ( $\mathrm{n}: 10)$.

III. Controls (CTR) ( $\mathrm{n}: 10)$ matched for age, sex, sexual hormonal status, BMI and cardiometabolic profile.

We evaluated:

In vivo:

Cardiometabolic clinical and analytical profile, endothelial dysfunction (FMD) and body composition (by DEXA).

\section{In vitro:}

Endothelial cells (EC) were exposed for $24 \mathrm{hs}$ or have been cultured for 7 days with the differents sera (groups I, II, III) to evaluate the inflammatory EC response (VCAM, ICAM, NfKB) and the reactivity (VWF) of the extracellular matrix (ECM).

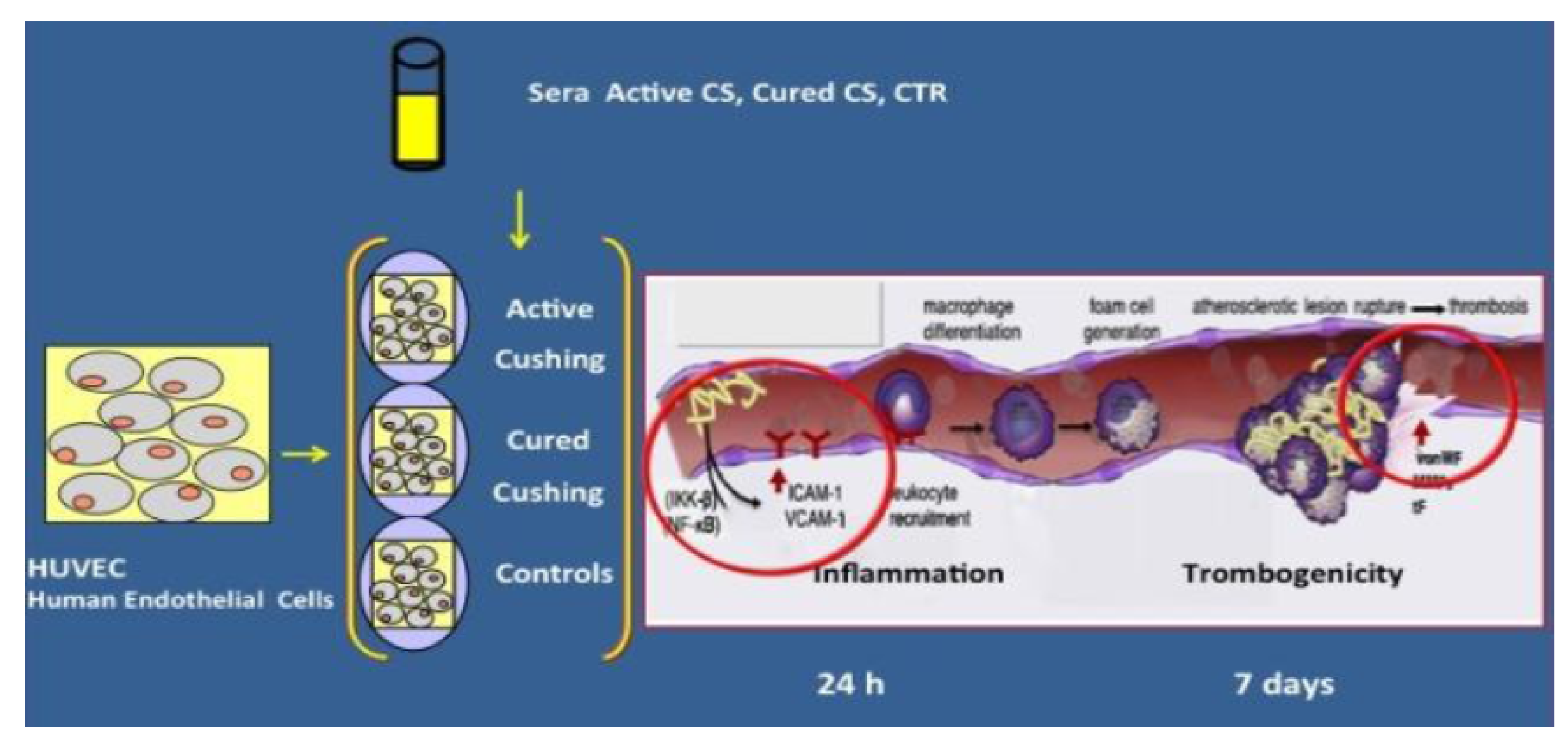

\begin{tabular}{|c|c|c|c|c|c|c|c|c|c|}
\hline \multirow[b]{2}{*}{$\begin{array}{c}\text { Baseline } \\
\text { Paramete } \\
\text { rs }\end{array}$} & \multicolumn{7}{|c|}{ Results } & \multirow[b]{2}{*}{ CTR (n:10) } & \multirow[b]{2}{*}{ P value } \\
\hline & $\begin{array}{c}\text { ACS } \\
(n: 10)\end{array}$ & $\begin{array}{c}\text { CCS } \\
(n: 10)\end{array}$ & $\begin{array}{c}\text { CTR } \\
\text { (n:10) }\end{array}$ & $P$ value & $\begin{array}{l}\text { Metabolic } \\
\text { parameters }\end{array}$ & ACS $(n: 10)$ & $\operatorname{CCS}(n: 10)$ & & \\
\hline \begin{tabular}{l}
\multicolumn{1}{c}{ rs } \\
Age \\
(vears)
\end{tabular} & $37.8 \pm 5.7$ & $37.3 \pm 6.7$ & $38.3 \pm 6.4$ & 0.947 & $\begin{array}{l}\text { Glucose } \\
\text { (mg/dl) }\end{array}$ & $103.0 \pm 22.5$ & $88.3 \pm 7.6$ & $96.0 \pm 11.6$ & 0.220 \\
\hline $\operatorname{Sex}(M / F)$ & $2 / 8$ & $2 / 8$ & $2 / 8$ & 1 & $\begin{array}{l}\text { HbA1C } \\
\text { (DCCT) }\end{array}$ & $6.2 \pm 0.8$ & $4.9 \pm 0.1$ & $5.4 \pm 0.3$ & 0.009 \\
\hline BMI & $26.5 \pm 2.1$ & $26.7 \pm 3.5$ & $25.6 \pm 3.7$ & 0.761 & $\mathrm{TC}(\mathrm{mg} / \mathrm{dl})$ & $182.1 \pm 31.8$ & $189.4 \pm 26.1$ & $176.0 \pm 18.5$ & 0.653 \\
\hline$(\mathrm{Kg} / \mathrm{m} 2)$ & & & & & LDL (mg/dl) & $105.1 \pm 24.1$ & $117.0 \pm 23.0$ & $98.5 \pm 23.9$ & 0.370 \\
\hline WC (cm) & $91.5 \pm 13.8$ & $87.1 \pm 13.9$ & $81.6 \pm 12.3$ & 0.390 & $\mathrm{HDL}$ (mg/dl & $54.4 \pm 11.6$ & $55.8 \pm 13.4$ & $70.6 \pm 25.8$ & 0.169 \\
\hline Ratio WH & $0.9 \pm 0.1$ & $0.8 \pm 0.1$ & $0.7 \pm 0.1$ & 0.447 & $\begin{array}{l}\text { ( } \mathrm{TG}(\mathrm{mg} / \mathrm{dl}) \\
\text { lo }\end{array}$ & $102.3 \pm 51.7$ & $92.5 \pm 36.8$ & $86.0 \pm 53.7$ & 0.788 \\
\hline $\begin{array}{l}\text { T2DM } \\
\text { (\%) } \\
\text { HTA (\%) }\end{array}$ & $2(6.6)$ & $2(6.6)$ & $0 \%$ & 0.159 & $\begin{array}{l}\text { UsRCP } \\
(\mathrm{mg} / \mathrm{dl})\end{array}$ & $0.22 \pm 0.09$ & $0.11 \pm 0.04$ & $0.08 \pm 0.07$ & 0.001 \\
\hline DLP (\%) & $\begin{array}{l}4(13.3) \\
1(3.3)\end{array}$ & $\begin{array}{l}2(0.0) \\
1(3.3)\end{array}$ & $0 \%$ & 0.501 & $\begin{array}{l}\text { Leucocytes } \\
\text { (mm3) }\end{array}$ & $\begin{array}{c}8590.0 \pm 147 \\
5.3\end{array}$ & $\begin{array}{c}6857.1 \pm 140 \\
7.0\end{array}$ & $\begin{array}{c}5922.8 \pm 149 \\
7.5\end{array}$ & 0.004 \\
\hline
\end{tabular}
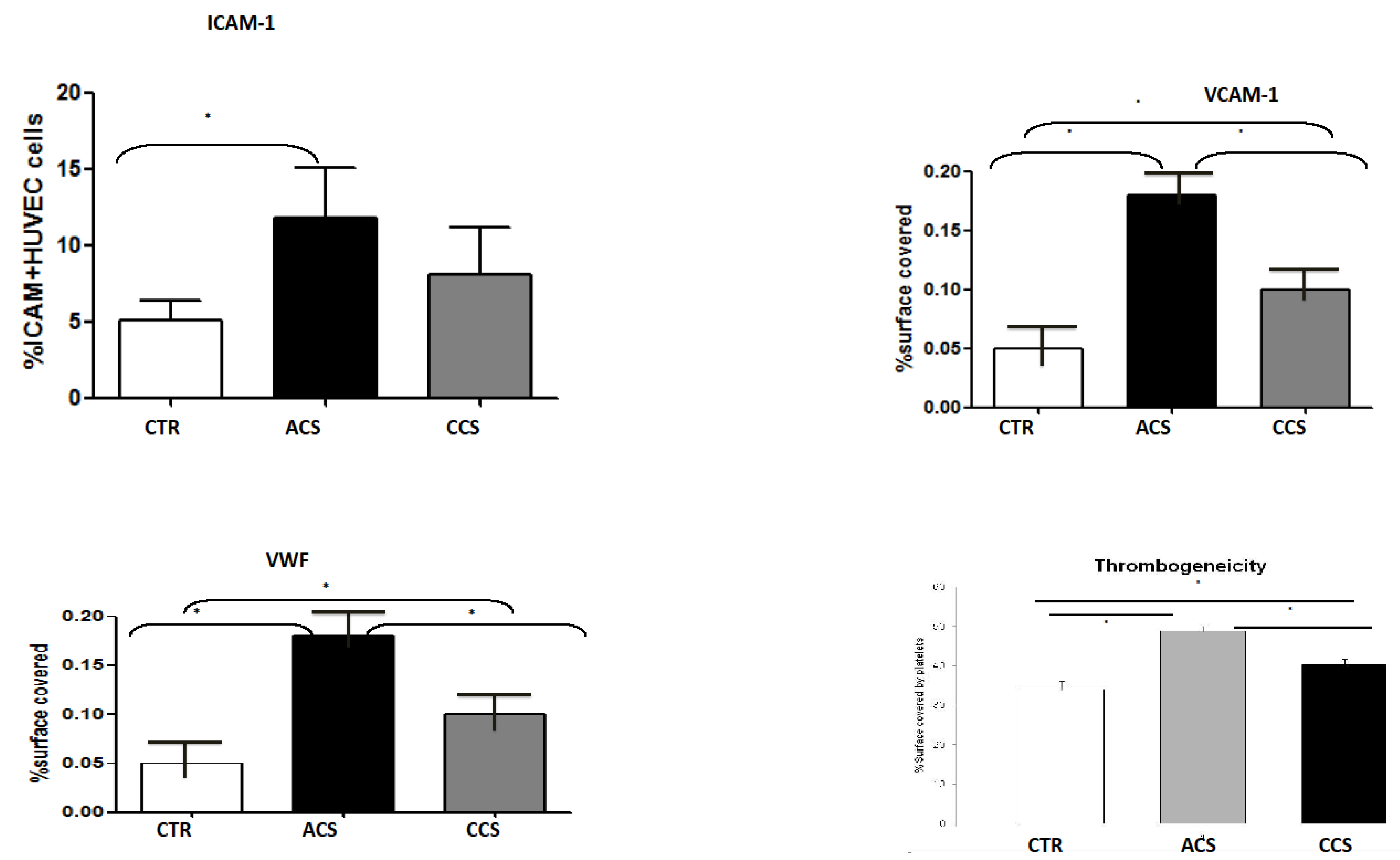

ACS patients have a clinical, subclinical and in vitro proatherothrombotic phenotype. EC exposed to CCS sera displayed augmented expression of ICAM $1(p=0.06), \operatorname{VCAM}(p=0.05)$, a higher synthesis of sub-endothelial VWF $(p=0.03)$ and higner EC reactivity towards circulating platelets: platelets adhesion than CTRs of the same sex, age and BMI $(p=0.04)$. NFkB is activated downstream.

\section{Conclusions}

This is the first translational study demostrating that the sera of patients with CCS have a deleterious atherothrombotic NFkB dependent potency on the endothelium inducing endothelial inflammatory activation and increased thrombogenicity. 